A. Albu-Rghaif and I. A. Lami, "DCSR: A Dynamic channel and resolution sampling for a Compressive Sensing Receiver to acquire GPS signals," presented at the 2013 IEEE International Conference on Microwaves, Communications, Antennas and Electronics Systems (COMCAS), Tel Aviv, Israel, 2013.

(C) 2013 IEEE. Personal use of this material is permitted. Permission from IEEE must be obtained for all other uses, in any current or future media, including reprinting/republishing this material for advertising or promotional purposes, creating new collective works, for resale or redistribution to servers or lists, or reuse of any copyrighted component of this work in other works. 


\title{
DCSR: A Dynamic channel and resolution sampling for a Compressive Sensing Receiver to acquire GPS signals
}

\author{
Ali Albu-Rghaif and Ihsan Alshahib Lami \\ Applied Computing Department \\ The University of Buckingham, UK \\ First.last@buckingham.ac.uk
}

\begin{abstract}
The use of Compressive Sensing (CS) technique to acquire GPS signals saves processing time when compared with software FFT based receivers. The computational complexity is reduced by up to $60 \%$. Such CS solution uses a fixed size measurement matrix chosen to offer a compromise between the processing overhead and signals acquisition success level. This paper proposes a dynamic acquisition algorithm for GPS signals based on CS. Instead of using a fixed size sampling channels and fixed correlators in the measurement matrix, our DSCR dynamically changes the number and size of the required Channels/Correlators according to the received GPS signals power during acquisition. This adaptive solution offers better fix capability when the GPS receiver is located in harsh signal environment or it will save valuable processing/decoding time (battery power, especially for Smartphones) when the receiver outdoors. A feedback loop is devised to control the sampling channels number and resize the measurement matrix.
\end{abstract}

Keywords- GPS signal; dynamic acquisition; sub-Nyquist sampling; compressive sensing

\section{INTRODUCTION}

Nowadays, GPS receivers are onboard all Smartphones. GPS/GNSS signals acquisition process is the most important and power/processing intensive in any GNSS solution, especially if the GNSS receiver to achieve short time to first fix. In time domain solutions, typical receivers have hundreds of hardware correlators' engine, while in the frequency domain, a high performance processor is needed to perform FFT/IFFT processes. Either way, the acquisition process requires huge overhead from the Smartphone's CPU and battery power. Acquisition becomes a drain especially during cold start and in harsh signal environments such as urban areas and indoors. Such GPS conventional receivers, for example, generate a replica SV signals PRN codes and carrier frequency with a range of Doppler frequencies $( \pm 4 \mathrm{KHz})$ to acquire the GPS signals. i.e. Signal acquisition is to find the correct code delay and frequency in the received signal. The search process is conducted in two dimensions; code phase search and Doppler shift search as shown in Fig. 1, i.e. each cell comprises a replica PRN code and locally generated frequency. The signal detection is achieved when the two parameters of Code and Doppler have high correlation value.

In order to achieve faster acquisition and low power consumption, $\mathrm{CS}$ is a more appropriate technique. It samples the received signal at low rate (below Nyquist rate) and downsizes the required number of correlators to acquire the signals [1], using the compressive multichannel sampling (CMS) technique.

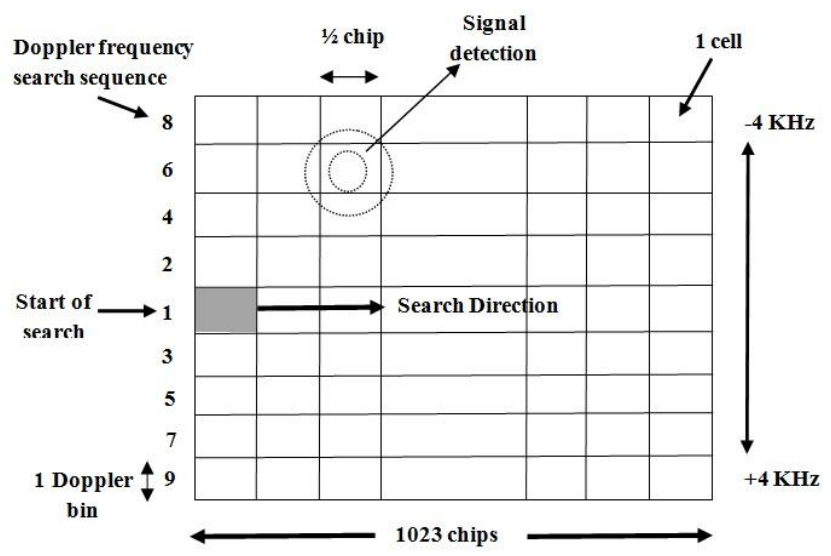

Fig. 1. Two dimension signal detection

Our proposed DCSR acquisition changes the number of the required channels/correlators according to the received signals power. We use the sub-Nyquist sampling technique on the received signals (GPS signals are used for testing our DCSR). Since the sampling rate depends on the information band (2.046 MHz), then a Continuous To Finite (CTF) block is used to convert the infinity measurement vector to multimeasurement vector. This reduces the computation from $\mathrm{N}$ (the actual dimension) to $\mathrm{M}$ (number of measurement) size problem, where $\mathrm{M}<<\mathrm{N}$ and also allow us to perform support recovery. Therefore, a feedback loop is designed to control the number of channels and resize the measurement matrix according to the received signal power; this means that when the signal is good, our algorithm shall greatly reduces the processing power while maintaining the fix rate of all available SV's signals. However, our algorithm deploys extra processing power when the signal power is weak resulting in maintaining the lock to the acquired SV's signals and thus maintaining the fix instead of giving up. Note that our DCSR algorithm performance has been compared to that achieved by the CMS algorithm. 
In the following sections, we first summarise related work based on the compressive sensing technique. Section III shows the mathematical model of the GPS signals. Section IV describes the DCSR for acquiring GPS signals. Section V explains the signal scenarios that have been tested with our proposed model and discusses the results. Section VI concludes this proposal and our future work.

\section{RELATED WORK}

Location based services/applications on Smartphones needs fast GPS acquisition with low power consumption. GPS receiver solutions based on CS can meet these requirements due to using sampling frequency below the Nyquist frequency and so perform fewer measurements than conventional algorithms.

Any RF signal can be recovered using CS if this signal has a sparse representation or it is relatively sparse [2]. A random demodulation (RD) technique has been designed to exploit the sparsity in the signal by mixing the received signal with a square $( \pm 1)$ waveform then samples the mixed signal at low rate to generate a sparse vector. This vector can now be easily evaluated in the digital processing domain [3]. Aimed at recent wireless communication technologies signals, an RD technique has been successfully applied to demodulate the direct sequence spread spectrum signals, where the input signal is mixed with PRN sequences at period equal to the Nyquist rate [4]. An enhanced solution has been achieved by using the subNyquist sampling technique [5]. This has overcome the following limitations of the RD approach: 1) the front-end in the sub-Nyquist technique became more flexible by choosing arbitrary periodic waveform rather than using a specific square waveform, 2) the rate of the periodic waveform is now equal to the sampling frequency, which is below the Nyquist rate, in contrast to the PRN sequences rate used in the RD, and 3) using a fixed number of channels became more reliable to recover the sparse signal. A combined RD with the subNyquist sampling technique is therefore more efficient to reconstruct the signal. i.e. This algorithm takes the advantages of sub-Nyquist method and using multichannel to sample the input signal at low rate, based on the RD concept [6].

In the same vein, a compressive multiplexer can be used to sample the RF signal below the Nyquist rate [7]. The main differences between this algorithm and the RD technique are; 1) this algorithm uses multichannel, 2) each of the channels will down convert the received signal to baseband, and then mix the baseband signal with a square wave at a period equal to the Nyquist rate, and 3) the output from the mixing process is summed once per chip before being sampled at low rate.

Solution that is based on CS, called CMS [1], samples the GPS signal via multichannel process to generate a sparse vector. The sparse vector is then digitally processed using CTF block and an Orthogonal Matching Pursuit (OMP) algorithm is used to find the recovery support. The recovery support represents the code phase-delay and the Doppler-shift of each acquired satellite signal. This algorithm samples the GPS signal based on the information rate, but it deploy a complicated hardware filter. Another CS approach that targets the GPS signal, called GCS [8], uses a low sampling frequency, and then correlates the sampled signal with a transform matrix, which represents a bank-correlators or parallel-correlators. Identifying the code phase delay and Doppler shift of the GPS signal is then obtained by two stages using deterministic sensing matrix (Walsh-Hadamard matrix) and the measurement matrix. The main differences that the CMS algorithm has over the GCS algorithms are: 1) it needs less computational complexity, 2) it requires more memory and 3 ) it has higher acquisition sensitivity due to testing only 1 bit length (20 C/A codes) of the GPS signal.

Using the same concept of GCS, but with incorporating the measurement matrix, introduces an indicator that distinguishes the type of modulation needed to acquire a BPSK signal or a BOC signal [9]. However, this approach does work for the signals that uses $\mathrm{BOC}(1,1)$ but it will not work with the current signals that use BOC $(1,1)$ like GPS L1-C, and Galileo E1-OS because these signals have different code length (10 ms for L1$\mathrm{C}$ and $4 \mathrm{~ms}$ for E1-OS).

The two CMS and GCS methods of acquiring the GPS signals based on CS indeed have achieved less computational complexity than the traditional acquisition methods. Our DCSR will overcome the drawback in these two methods by maintaining the "Sub-Nyquist Sampling" but use an adaptive/dynamic algorithm that will change the number and size of the required Channels/Correlators according to the acquired GPS signals power.

\section{GPS SIGNAL STRUCTURE}

The GPS L1-C/A signal is based on direct sequence spread spectrum. The navigation messages with 50 bit per second (1 bit every $20 \mathrm{~ms}$ ) are spread by the C/A code using the Code Division Multiple Access technique. This code is 1023 chips long and is repeated every $1 \mathrm{~ms}$ at a rate of $1.023 \mathrm{MHz}$ (i.e. 20 $\mathrm{C} / \mathrm{A}$ codes are used to spread a single bit of the navigation message). Each GPS satellite has a unique C/A code out of 32 possible codes chosen to be almost orthogonal to each other. The spreading sequences are BPSK modulated with L1 carrier frequency that is centred at $1575.42 \mathrm{MHz}$ and the mathematical representation of the GPS signals is shown in (1):

$$
S^{k}(t)=\sum \sqrt{2 P}\left[C_{k}(t) D_{k}(t) \cos \left(2 \pi F_{L 1} t\right)\right]
$$

Where $S^{k}(\mathrm{t})$ represents the signals transmitted from $\mathrm{k}^{\text {th }}$ satellite $(\mathrm{k}=1 \ldots 32), \mathrm{P}$ is the power of the transmitted signal, $\mathrm{C}_{\mathrm{k}}(\mathrm{t})$ is the C\A code assigned to the $\mathrm{k}$ satellite, $\mathrm{D}_{\mathrm{k}}(\mathrm{t})$ is the navigation data sequence and $\mathrm{F}_{\mathrm{L} 1}$ is the carrier frequency of L1.

The minimum GPS L1-C/A received power measured by 3 $\mathrm{dBi}$ linearly polarized user antenna is $-158.5 \mathrm{dBW}$. The GPS signal reaches any receiver's antenna either directly (line of sight signal) or indirectly (multipath signal). The received multipath GPS signals from different satellites can be expresses as:

$$
r(t)=\sum_{l=1}^{L} \sum_{m=1}^{M} A C_{l, m}\left(t-n T_{c}-\tau\right) D_{l, m}(t-\tau) e^{j\left(2 \pi F_{d_{l, m}} t+\theta_{l, m}\right)}+w(t)
$$

Where $\quad r(t), A, \tau, T_{c}, F_{d}, \theta, L, M$ and $w(t)$ represent the received signal, power of received signal, code phase delay (caused by ionosphere, troposphere and the multipath effects), 
chip period $(1 / 1.023 \mathrm{MHz})$, carrier frequency with Doppler shift, carrier phase, LOS signals from $l=1$ to $L$, multipath signals from $m=1$ to $M$ and the complex AWGN respectively.

\section{THE DCSR}

$\mathrm{CS}$ is guaranteed to acquire and reconstruct any sparse signal with much less measurements, by exploiting the sparsity in the signal [10]. CS samples the received signal according to either the "occupation information band" or the "information rate", which is less than the minimum Nyquist sampling rate of double the information bandwidth. In particular, the coherence $\mu(\Phi, \Psi)$ between the sensing matrix $\varphi$ and the transformation matrix $\psi$ should be low and $\mu(\Phi, \Psi) \in[1, \sqrt{n}]$ so to enable accurate recovery of the compressed signal [2].

$$
\mu(\Phi, \Psi)=\sqrt{n} \max _{l \leq k, m \leq n}\left|\left\langle\varphi_{k}, \psi_{m}\right\rangle\right| \text { and } \mathrm{k}<m \ll n
$$

The deterministic sensing matrix will downsize the number of the required measurement. Nonetheless, random sensing matrix such as Gaussian matrix, binary matrix [1] or Bernoulli matrix [8] can also be used as they would satisfy the Restricted Isometry Property that depends on the orthogonality between the arbitrary column vectors [11].

\section{A. Sampling and Sensing}

Actually, the sub-Nyquist sampling technique is proven to be more reliable to sample signals as well as being easy to implement [12], because it produces a compressed signal that is suitable to be used with the CS technique. Therefore, to achieve the desired acquisition accuracy, this technique has been adopted in our proposal algorithm to sample and sense the received GPS signal, as shown in Fig. 2.

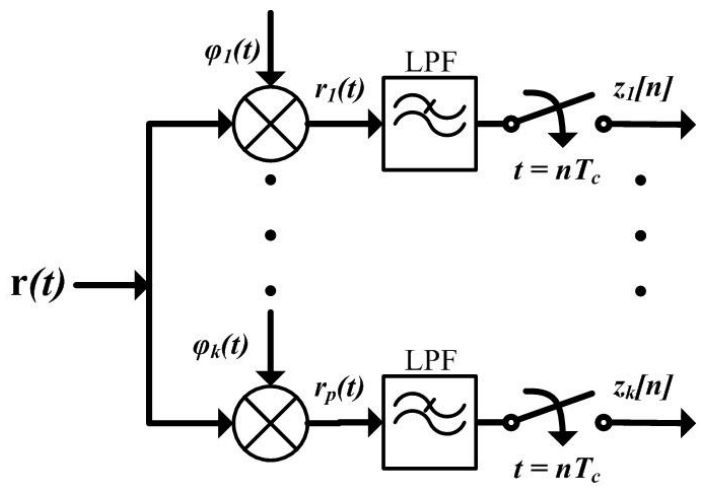

Fig. 2. Sub-Nyquist multi-channel sampling

The received GPS signals are multiplied by the square periodic waveform $\varphi_{\mathrm{k}}(\mathrm{t})$ using a number of channels simultaneously; these waveforms are equivalent to the binary sensing matrix that will be used, together with the bankcorrelators matrix $\beta$, to acquire the GPS signal. The rate of the square wave $\left(F_{p}\right)$ is equal to the sampling rate $\left(F_{s}\right)$, which corresponds to the bandwidth of the received signal, where $\left(\mathrm{F}_{\mathrm{p}}\right.$ $=\mathrm{F}_{\mathrm{s}} \approx$ Bandwidth) [5]. The sub-Nyquist algorithm samples the received signals according to the maximum bandwidth of the multiband signals. In our case only the GPS signals are tested, and so the sample rate is $2.046 \mathrm{MHz}$. The mixing of the periodic waveform with the GPS signal will alias the spectrum of the GPS signal to the baseband, and so the waveform rate becomes related to the signal's bandwidth. i.e., the output will becomes a linear combination of the frequency shift copies of $F_{p}$. The baseband signals are then filtered by using low-pass filters and the output are sampled at low rate corresponding to the signal bandwidth.

$$
Z_{i}(\omega)=\sum_{k \in K} \phi_{i}\left(k \omega_{p}\right) R_{i}(\omega), \quad \omega \epsilon \omega_{s}
$$

\section{B. Acquiring GPS signal}

In order to acquire the GPS signals, we first constructs the frame $\mathrm{F}$ from the jointly sparse over time vectors $\mathrm{z}[\mathrm{n}]$ in $(5 \&$ 6) using the CTF block, as shown in Fig. 3. The decomposition of the $\mathrm{F}$ frame is optional to remove the noise space, where $\mathrm{V}$ is prepared by taking the Eigen-vectors of F multiplied by the square-root of the Eigen-values (7).

$$
\begin{aligned}
& z[n]=\left[z_{l}[n], \ldots, z_{k}[n]\right]^{T} \\
& F=\sum_{n} z[n] z^{H}[n] \\
& F=V V^{H}
\end{aligned}
$$

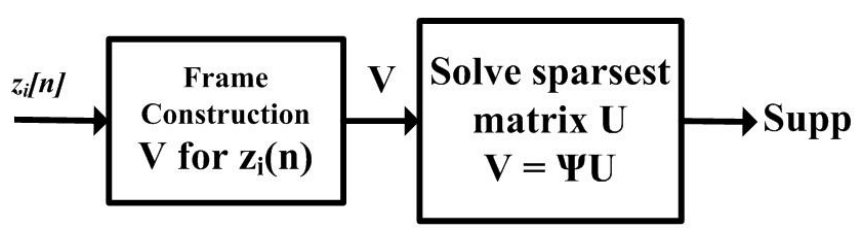

Fig. 3. Continuous to finite block

The CTF block converts the infinity measurement vector to multi-measurement vector. The sparse matrix $U$ (8) can be solved using the "reduce and boost" algorithm that reduces the problem from multi-measurement vector to a single measurement vector, and using the "pursuit algorithm" such as the "orthogonal matching pursuit algorithm" to find the support values of the sparse matrix [13].

$$
V=\Psi U
$$

Where $\Psi$ is the measurement matrix $\Psi(\omega) \triangleq\left[\psi_{1}(\omega), \ldots, \psi_{k}(\omega)\right]^{T}$, which and consists from the sensing matrix $\phi(\omega)$ and the bank-correlators matrix $\beta(\omega)$. The measurement matrix can be expressed as:

$$
\psi_{k,[l, p, q]}(t)=\sum_{l=1}^{L} \sum_{p \in P} \sum_{q \in Q} \varphi_{k}(t) \beta_{l, p, q}(t)
$$

Where P and Q are the "search step of code phase delay" (at half chip resolution) and the "Doppler frequency shift" at 500 $\mathrm{Hz}$ steps respectively.

$$
[\beta]_{l, p, q}=C_{l}\left(t-p T_{c}\right) e^{j\left(2 \pi\left(F_{d}+q\right) T_{c}\right.}
$$

The bank-correlators matrix is the $\mathrm{L}|\mathrm{P} \| \mathrm{Q}|$ columns vectors, where each column represents an expected shift of the code phase delay with Doppler shift of each GPS satellite signal. After the completion of the support recovery of the sparse matrix U, the right code phase delay and Doppler frequency 
shift of acquired satellites $\left(\operatorname{supp}_{l, p, q}\right)$ are determined by calculating the maximums values of the column vectors of $U$ $(S=\operatorname{supp}(U))$.

\section{Dynamism}

Acquiring GPS signals based CMS algorithm suffers from high processing requirements if to acquire signals in harsh environment, else GPS signals lock is lost. i.e. Using higher number of channels will produce higher rate of acquired satellites signals, but that means these channels will has to be used in good signal areas too, which is a waste of efforts. Our proposed algorithm is to overcome this drawback of using a fixed number of channels by controlling the deployed number of channels and size of used correlations on the go as needed determined by the actual signals strength.

In fact, the DCSR algorithm will resize both sensing and measurement matrices by using the feedback control as shown in Fig. 4. The feedback-controller continuously measures the power of the received signal to determine the signal complexity level. The received power is calculated as:

$$
P_{r}=P_{t} G_{t} G_{a} / 4 \pi \rho^{2}
$$

where $P_{r}, P_{t}, G_{t}, G_{a}, \rho$ are the received GPS signal power, transmit power, satellite antenna gain (10.2 to $12.3 \mathrm{~dB})$, effective area of receiver antenna (12) and measured pseudoranges respectively [14].

$$
G_{a}=\lambda^{2} / 4 \pi \quad \lambda \text { is the wavelength }=0.1903 \mathrm{~m}
$$

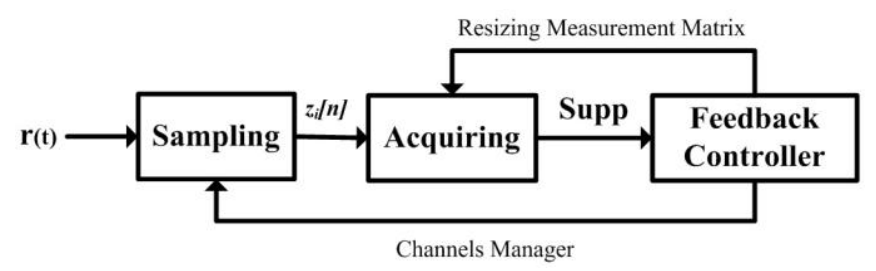

Fig. 4. Dynamic GPS signal acquisition

Once the power of the GPS signals are calculated, we easily determine the carrier to noise ratio $(\mathrm{C} / \mathrm{N})$ or signal to noise ratio (SNR) [15].

$$
C / N_{o}=P_{r(d B W)}-N_{T H}
$$

Where $C / N_{o}$ is the nominal carrier to noise ratio in $\mathrm{dBW}$, $P_{r(d B W)}$ is the receive power in $\mathrm{dBW}\left(\operatorname{lolog}\left(P_{r}\right.\right.$ in $\left.\left.W\right)\right)$ and $N_{T H}$ is the thermal noise power $(-204 \mathrm{dBW})$.

$$
C / N=C / N_{o}-N_{F}
$$

Where $N_{F}$ is the cascade Noise Figure of this receiver.

The range of the SNR values of the received signals depend on the front-end bandwidth $\mathrm{BW}(\mathrm{dBW})\left(\mathrm{BW}=10 \log \left(\mathrm{F}_{\mathrm{B}}\right)\right)$, and the $\mathrm{F}_{\mathrm{B}}$ is filter bandwidth:

$$
S N R=C / N-B W
$$

More precisely, our dynamic design is based on three levels of measurement complexity. High measurements level is used in bad reception areas and so 600 channels are selected to compensate for the sensing and measurements for these weak signals. While when outdoors, our simulations show that using only 240 channels are sufficient to acquire the signals (represents a low measurements level). Finally, 480 channels are chosen as a middle case to help compare the DCSR and CMS algorithms performance. Our simulations show that these three channel-number selection-levels make the DSCR much more efficient than the CMS by using only necessary resources depending on the reception environment.

Furthermore, the design of the measurement matrix $\psi_{k,[l, p, q]}(t)$ makes the algorithm more powerful because each element in the measurement matrix contains all information of a single satellite (9), in contrast to the design of the measurement matrix in CMS. So, when the number of channels is increased/decreased, the information of the satellite in the measurement matrix does not get not affected, and only the sensing property in the measurement matrix will be changed.

\section{Simulations Results}

The DCSR algorithm performance has been compared with that used by the CMS algorithm. our dynamic scenario for the GPS signal, that has $\mathrm{C} / \mathrm{N}$ value varies according to the signals environment, is listed in table I. (Note that the $\mathrm{C} / \mathrm{N}$ is varied from $50 \mathrm{~dB}-\mathrm{Hz}$ to $25 \mathrm{~dB}-\mathrm{Hz}$, the bandwidth of low-pass filter is set at $2 \mathrm{MHz}$ with a $3 \mathrm{~dB}$ cascade noise figure, and the $\mathrm{C} / \mathrm{N}$ values corresponds to -5 to $-30 \mathrm{~dB}$ SNR values).

The scenario in table I start with an open sky reception area having Line Of Sight (LOS) signals only (S1). These signals are then degraded and multipath signals are added to the existing LOS signals. We consider two signal degradations as shown in (S2) \& (SM). The urban area scenario is represented by only multipath signals (M). Since the algorithms are based on acquiring weak GPS signal, the length of tested signals is chosen to be $20 \mathrm{~ms}$. This will increase the acquisition sensitivity by around $13 \mathrm{~dB}$ more than the normal methods ( $1 \mathrm{~ms}$ length), where the gain obtained by the $20 \mathrm{~ms}$ acquisition is around $46 \mathrm{~dB}$ compared with the $33 \mathrm{~dB}$ gain obtained from the $1 \mathrm{~ms}$ processing $\left(\mathrm{G}_{\mathrm{dB}}=20 \log \sqrt{\mathrm{n}}\right)$.

The bank-correlators matrix has a half chip resolution for code phase delay; $500 \mathrm{~Hz}$ frequency search steps with Doppler frequency range $\pm 4 \mathrm{KHz}$, and the maximum channel delay distribution of the $\mathrm{C} / \mathrm{A}$ code is $30 \mathrm{~T}_{\mathrm{c}}$ to reduce the implementation complexity. Additionally, to reduce the computational complexity as much as possible, the "approximate conjugate direction gradient pursuit" algorithm [16] is used rather than the "orthogonal matching pursuit" algorithm. Our simulations show that both algorithms have the same performance over all signal conditions.

TABLE I. OPEN-SKY AND MULTIPATH SCENARIOS

\begin{tabular}{|c|l|c|}
\hline \multirow{2}{*}{ CIN dB-Hz } & \multicolumn{3}{|c|}{ LOS \& Multipath signals Scenarios } \\
\cline { 2 - 3 } Number of received signals & Scenario name \\
\hline 50 & 5 LOS signals & $\mathrm{S} 1$ \\
\hline 45 to 40 & $\begin{array}{l}5 \text { LOS signals and one } \\
\text { multipath for each signal }\end{array}$ & $\mathrm{S} 2$ \\
\hline 35 & $\begin{array}{l}5 \text { LOS signals and two } \\
\text { multipath for each signal }\end{array}$ & SM \\
\hline 30 to 25 & $\begin{array}{l}\text { 15 multipath signals for 5 } \\
\text { satellites }\end{array}$ \\
\hline
\end{tabular}


The simulated dynamic scenario is illustrated in Fig. 5-a, where the $\mathrm{C} / \mathrm{N}$ values represent the various received signal conditions. The dynamic scenario is changed gradually to simulate Smartphone movements. The results shows that the number of acquired satellites signals by the DCSR algorithm is almost constant at all signal strength cases, while the CMS algorithm has failed to acquire signals in low signal conditions as shown in Fig. 5-b.

Fig. 5-c shows how the DCSR has dynamically switched the number of channels as dictated by the received signal condition. So, in order to acquire the GPS signals in harsh environment, the DCSR switches to the maximum number of channels, while in ideal environment "open-sky", the DCSR uses the minimum number of channels to save power and reduce processing time.

The results therefore show the adaptability of the DCSR to be work in various environments to maintain the performance of the GPS receiver while saving processing and battery power.
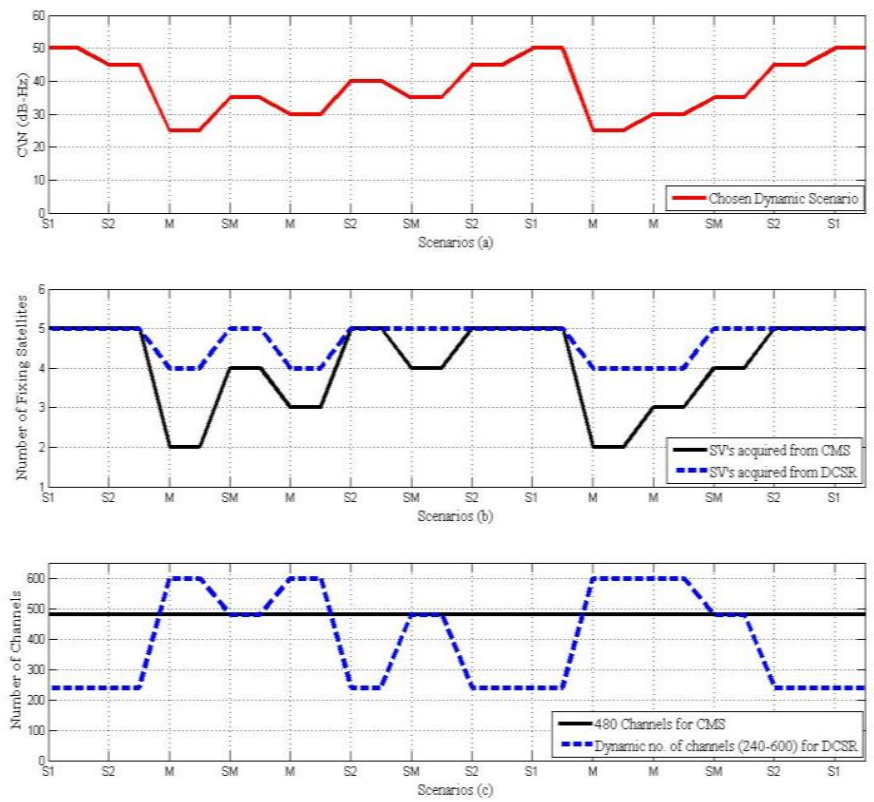

Fig. 5. Dynamic channels vs. fix number of channel performance, (a) the dynamic scenario, (b) number of satellite signals acquired from CMS \& DCSR algorithms and (c) number of channels of CMS \& DCSR algorithms

\section{CONCLUSION}

The DCSR algorithm can reduce processing time and so minimise the power consumption required by a GPS receiver to acquire signals in outdoors. This is accomplished by dynamically altering the number of measurements and the required number of sparse channels to suit the actual signal strength. Also, the DCSR maintains lock to the available signals in difficult signal conditions by using extra number of channels to compensate the measurements. Using the subNyquist sampling technique does simplify the receiver frontend and allow sampling the GPS signals at low rate. Finally, applying the "gradient pursuit algorithm" to recover the sparse signal will also reduce the computational complexity.

\section{ACKNOWLEDGMENT}

This research is supported by a government scholarship from Iraqi ministry of higher education.

\section{REFERENCES}

[1] X. Li, Y. C. Eldar and A. Scaglione, "Low complexity acquisition of GPS signals," in Signal Processing Advances in Wireless Communications (SPAWC), 2011 IEEE 12th International Workshop on, 2011.

[2] E. J. Candes and M. B. Wakin, "An introduction to compressive sampling," Signal Processing Magazine, IEEE, vol. 25, no. 2, pp. 21-30, 2008.

[3] S. Kirolos, J. Laska, M. Wakin, M. Duarte, D. Baron, T. Ragheb, Y. Massoud and R. Baraniuk, "Analog-to-information conversion via random demodulation," in Design, Applications, Integration and Software, 2006 IEEE Dallas/CAS Workshop on, 2006.

[4] K. Fyhn, T. Arildsen, T. Larsen and S. H. Jensen, "Demodulation of a Subsampled Direct Sequence Spread Spectrum Signal using Compressive Signal Processing," arXiv preprint arXiv:1110.5176, 2011.

[5] M. Mishali and Y. C. Eldar, "From theory to practice: Sub-Nyquist sampling of sparse wideband analog signals," Selected Topics in Signal Processing, IEEE Journal of, vol. 4, no. 2, pp. 375-391, 2010.

[6] J. Yoo, S. Becker, M. Monge, M. Loh, E. Candes and A. EmamiNeyestanak, "Design and implementation of a fully integrated compressed-sensing signal acquisition system," in Acoustics, Speech and Signal Processing (ICASSP), 2012 IEEE International Conference on, 2012.

[7] J. Slavinsky, J. N. Laska, M. A. Davenport and R. G. Baraniuk, "The compressive multiplexer for multi-channel compressive sensing," in Acoustics, Speech and Signal Processing (ICASSP), 2011 IEEE International Conference on, 2011.

[8] S. Kong, "A Compressed Sensing Technique for GNSS Signal Acquisition," in Proceedings of the 2012 International Technical Meeting of The Institute of Navigation, 2012.

[9] S.-H. Kong, "A Deterministic Compressed GNSS Acquisition Technique," 2013.

[10] D. L. Donoho, "Compressed sensing," Information Theory, IEEE Transactions on, vol. 52, no. 4, pp. 1289-1306, 2006.

[11] E. J. Candes, "The restricted isometry property and its implications for compressed sensing," Comptes Rendus Mathematique, vol. 346, no. 9, pp. 589-592, 2008.

[12] M. Mishali, Y. C. Eldar, O. Dounaevsky and E. Shoshan, "Xampling: Analog to digital at sub-Nyquist rates," IET circuits, devices $\backslash \&$ systems, vol. 5, no. 1, pp. 8-20, 2011.

[13] M. Mishali and Y. C. Eldar, "Reduce and boost: Recovering arbitrary sets of jointly sparse vectors," Signal Processing, IEEE Transactions on, vol. 56, no. 10, pp. 4692-4702, 2008.

[14] F. S. T. V. Diggelen, A-GPS: Assisted GPS, GNSS, and SBAS, Artech House Publishers, 2009.

[15] A. Joseph, "GNSS Solutions: Measuring GNSS Signal Strength," Inside GNSS-Engineering Solutions for the Global Navigation Satellite System Community, vol. 5, no. 8, pp. 20-25, 2010.

[16] T. Blumensath and M. E. Davies, "Gradient pursuits," Signal Processing, IEEE Transactions on, vol. 56, no. 6, pp. 2370-2382, 2008. 